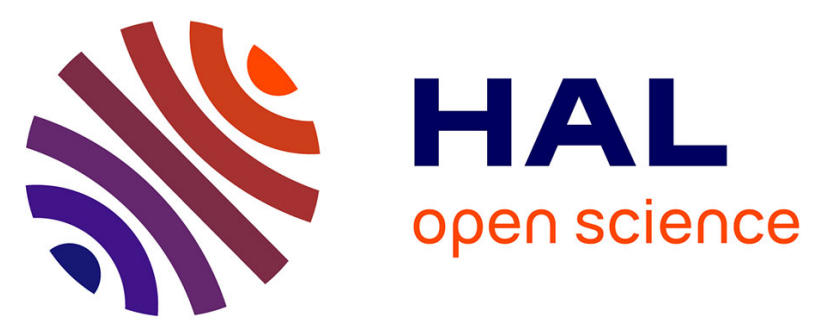

\title{
Robust vision-based robot localization using combinations of local feature region detectors
}

Arnau Ramisa, Adriana Tapus, David Aldavert, Ricardo Toledo, Ramon

Lopez de Mantaras

\section{To cite this version:}

Arnau Ramisa, Adriana Tapus, David Aldavert, Ricardo Toledo, Ramon Lopez de Mantaras. Robust vision-based robot localization using combinations of local feature region detectors. Autonomous Robots, 2009, 27 (4), pp.373-385. 10.1007/s10514-009-9136-9 . hal-00847376

\section{HAL Id: hal-00847376 \\ https://hal.science/hal-00847376}

Submitted on 23 Jul 2013

HAL is a multi-disciplinary open access archive for the deposit and dissemination of scientific research documents, whether they are published or not. The documents may come from teaching and research institutions in France or abroad, or from public or private research centers.
L'archive ouverte pluridisciplinaire HAL, est destinée au dépôt et à la diffusion de documents scientifiques de niveau recherche, publiés ou non, émanant des établissements d'enseignement et de recherche français ou étrangers, des laboratoires publics ou privés. 


\title{
Robust vision-based robot localization using combinations of local feature region detectors
}

\author{
Arnau Ramisa • Adriana Tapus · David Aldavert . \\ Ricardo Toledo $\cdot$ Ramon Lopez de Mantaras
}

Received: 31 January 2009 / Accepted: 5 August 2009 / Published online: 27 August 2009

(C) Springer Science+Business Media, LLC 2009

\begin{abstract}
This paper presents a vision-based approach for mobile robot localization. The model of the environment is topological. The new approach characterizes a place using a signature. This signature consists of a constellation of descriptors computed over different types of local affine covariant regions extracted from an omnidirectional image acquired rotating a standard camera with a pan-tilt unit. This type of representation permits a reliable and distinctive environment modelling. Our objectives were to validate the proposed method in indoor environments and, also, to find out if
\end{abstract}

This work was partially supported by the FI grant from the Generalitat de Catalunya, the European Social Fund, and the grant 2009-SGR-1434 and the MID-CBR project grant TIN2006-15140-C03-01 and FEDER funds and the grant 2005-SGR-00093 and the MIPRCV Consolider Ingenio 2010.

A. Ramisa ( $\varangle) \cdot$ R. Lopez de Mantaras

Artificial Intelligence Research Institute (IIIA, CSIC),

UAB Campus, E-08193 Bellaterra, Barcelona, Spain

e-mail: aramisa@iiia.csic.es

R. Lopez de Mantaras

e-mail: mantaras@iiia.csic.es

A. Tapus

University of Southern California, Los Angeles, CA 90089, USA

Present address:

A. Tapus

ENSTA-ParisTech, Paris, France

e-mail: adriana.tapus@ieee.org

D. Aldavert $\cdot R$. Toledo

Computer Vision Center (CVC), UAB Campus,

E-08193 Bellaterra, Barcelona, Spain

D. Aldavert

e-mail: david.aldavert@cvc.uab.cat

R. Toledo

e-mail: ricardo.toledo@cvc.uab.cat the combination of complementary local feature region detectors improves the localization versus using a single region detector. Our experimental results show that if false matches are effectively rejected, the combination of different covariant affine region detectors increases notably the performance of the approach by combining the different strengths of the individual detectors. In order to reduce the localization time, two strategies are evaluated: re-ranking the map nodes using a global similarity measure and using standard perspective view field of $45^{\circ}$.

In order to systematically test topological localization methods, another contribution proposed in this work is a novel method to see the degradation in localization performance as the robot moves away from the point where the original signature was acquired. This allows to know the robustness of the proposed signature. In order for this to be effective, it must be done in several, variated, environments that test all the possible situations in which the robot may have to perform localization.

Keywords Topological localization - Vision based localization · Panoramic vision · Affine covariant region detectors

\section{Introduction}

Finding an efficient solution to the robot localization problem will have a tremendous impact on the manner in which robots are integrated into our daily lives. Most tasks for which robots are well suited demand a high degree of robustness in their localizing capabilities before they are actually applied in real-life scenarios (e.g., assistive tasks).

Since localization is a fundamental problem in mobile robotics, many methods have been developed and discussed in 
the literature. The existing approaches can be broadly classified into three major types: metric, topological and hybrid. Metric approaches (Dissanayake et al. 2001; Castellanos and Tardos 1999; Thrun 1998, 2000) are useful when it is necessary for the robot to know its location accurately in terms of metric coordinates (i.e. Cartesian coordinates). However, the state of the robot can also be represented in a more qualitative manner, by using a topological map (i.e. adjacency graph representation) (Choset and Nagatani 2001; Tapus and Siegwart 2006; Beeson et al. 2005). Because the odometry does not provide enough and complete data in order to localize a mobile autonomous robot, laser range finders and/or vision sensors are usually used to provide richer scene information. The rapid increase in computational power in the last few years had a significant impact in the development of better approaches to solve the simultaneous localization and mapping (SLAM) problem, by using qualitative information provided by vision. Furthermore, vision units are cheaper, smaller and more practical than large expensive laser scanners.

In this work, we propose a topological vision-based localization approach of a mobile robot evolving in dynamic indoor environments. Robot visual localization and place recognition are not easy tasks, and this is mainly due to the perceptive ambiguity of acquired data and the sensibility to noise and illumination variations of real world environments. We propose to approach this problem by using a combination of affine covariant detectors so as to extract a robust spatial signature of the environment.

We decided to use combinations of the following three feature region detectors: MSER (Maximally Stable Extremal Regions) (Matas et al. 2002), Harris-Affine (Lindeberg 1998), and Hessian-Affine (Mikolajczyk and Schmid 2004), which have shown to perform better when compared to other region detectors.

When a new signature is acquired, it is compared to the stored panoramas from the a priori map. The panorama with the highest number of matches is selected. To improve the results and discard false matches, the essential matrix is computed and the outliers filtered. Finally, the panorama with the highest number of inliers is selected as the best match. In our approach images are acquired using a rotating conventional perspective camera. When a set of images covering 360 degrees is acquired, they are projected to cylindrical coordinates and the feature regions are extracted and described. The descriptors constellation is next constructed automatically. Hence, by using feature regions to construct the signature of a location, our approach is much more robust to occlusions and partial changes in the image than the approaches using global descriptors. This robustness is obtained because many individual regions are used for every signature of a location and, thus, if some of them disappear the constellation can still be recognized.
Nevertheless, combining different region detectors increases the computational time and memory requirements. For this reason we show that a re-ranking mechanism based on a global appearance-based similarity measure can be used to prioritize the most similar map nodes.

This framework gives us an interesting solution to the perceptual aliasing problem (one of the main difficulties when dealing with qualitative navigation and localization). Our approach is validated in real world experiments and is compared with other vision-based localization methods.

Defining compact and efficient representations for places has a number of advantages, like minimizing the amount of memory used, reducing both the time employed exploring the environment to acquire a complete map and the search effort to perform localization afterwards.

Similar methods to the one proposed in this work, like that of Booij et al. (2007) or Valgren and Lilienthal (2008), usually first acquire an over-complete map of the environment and then memory usage is reduced using a technique like the Incremental Spectral Clustering to discard or group unnecessary nodes and reduce the final size of the map. Moreover, signatures with a higher degree of robustness to viewpoint change would also boost the positive effect of such techniques.

However, usually these types of signatures are evaluated in a success/failure fashion in complete navigation or localization tasks, without effectively comparing the advantages and drawbacks of the different alternatives that exist to construct them in a standardized way.

In consequence, another contribution of this work is a new evaluation methodology to assess how robust a global localization signature is regarding viewpoint change in different indoor environments.

The remainder of this paper is organized as follows: In Sect. 2 we will first present a review of most recent related work on visual-based localization and navigation. Section 3 summarizes the different affine covariant region detectors and descriptors that we used in our work. Section 4 describes the localization procedure in details and the experimental design. Experimental results are reported in Sect. 5. And finally, Sect. 6 concludes our paper.

\section{Related work}

Over the last decade, many appearance-based localization methods have been proposed (Owen and Nehmzow 1998; Franz et al. 1998; Se et al. 2002). SIFT (Scale Invariant Feature Transform) features (Lowe 2004) have been widely used for robot localization. The SIFT approach detects and extracts feature region descriptors that are invariant to illumination changes, image noise, rotation and scaling. In Se et al. (2002), the authors used SIFT scale and orientation 
constraints so as to match stereo images; least-square procedure was used to obtain better localization results. The model designed by Andreasson et al. (2005) combines the SIFT algorithm for image matching and Monte-Carlo localization; their approach takes the properties of panoramic images into consideration. Another interesting subset of invariant features are the affine covariant regions which can be correctly detected in a wide range of acquisition conditions (Mikolajczyk et al. 2005). Therefore, Silpa-Anan and Hartley (2004) construct an image map based on Harris Affine feature Regions with SIFT descriptors that is later used for robot localization.

The work proposed by Tapus and Siegwart (2006) defined fingerprints of places as generic descriptors of environment locations. Fingerprints of places are circular lists of features and they are represented as a sequence of characters where each character is an instance of a specific feature type. The author used a multi-perceptional system and global lowlevel features (i.e., vertical edges, color blobs, and corners) are employed for localization.

Moreover, recently, several robot global localization methods similar to the one proposed in this paper have been presented. Booij et al. (2007) build first an appearance graph from a set of training images recorded during exploration. The Differences of Gaussians (DoG) feature detector and the SIFT descriptor are used to find matches between omnidirectional images in the same manner as described in Lowe (2004), and the essential matrix relating every two images is computed with the 4-point algorithm with planar motion assumption in RANSAC. The similarity measure between each pair of nodes of the map is the ratio between the inliers according to the essential matrix and the lowest number of features found in the two images. Appearance based navigation is performed by first localizing the robot in the map with a newly acquired image and then using Dijkstra's algorithm to find a path to the destination. Several navigation runs are successfully completed in an indoor environment even with occlusions caused by people walking close to the robot. Valgren and Lilienthal (2008) evaluate an approach focusing on visual outdoor localization across seasons using spherical images taken with a high resolution omnidirectional camera. Then, Upright Speeded Up Robust Features (U-SURF) (Bay et al. 2008), that are not invariant to rotation, are used to find matches between the images and the 4-point algorithm is used to compute the essential matrix. Indoor localization differs from outdoor localization in that typically distances to objects and walls is much shorter, and therefore the appearance of objects changes faster if one moves away from a reference point. Furthermore, indoor locations tend to have few texture and repetitive structures that complicates the data association problem, but they are positively less affected by environmental changes (e.g., time of the day; seasons).
Cummins and Newman (2008) proposed an approach that uses a probabilistic bail-out condition based on concentration inequalities. They have applied the bail-out test to accelerate an appearance-only SLAM system. Their work has been extensively tested in outdoor environments. Furthermore, the work presented by Angeli et al. (2008) describes a new approach for global localization and loop detection based on the bag of words method.

\section{Affine covariant region detectors}

An essential part of our approach is the extraction of discriminative information from a panoramic image so it can be recognized later under different viewing conditions. This information is extracted from the panoramic image using affine covariant region detectors. These detectors find regions in the image that can be identified even under severe changes in the point of view, illumination, and/or noise.

Recently Mikolajczyk et al. (2005) reviewed the state of the art of affine covariant region detectors individually. In this review they concluded that using several region detectors at the same time could increase the number of matches and thus improve the results. Hence, based on their results, we have used all the combinations of the following three complementary affine covariant region detectors: (1) HarrisAffine, (2) Hessian-Affine, and (3) MSER (Maximally Stable Extremal Regions), so as to increase the number of detected features and thus of potential matches. Examples of detected regions for the three region detectors can be seen in Fig. 1. These three region detectors have a good repeatability rate, a reasonable computational cost and they are briefly detailed below.

1. The Harris-Affine detector is an improvement of the widely used Harris corner detector. It first detects Harris corners in the scale-space with automatic scale selection using the approach proposed by Lindeberg (1998), and then estimates an elliptical affine covariant region around the detected Harris corners. The Harris corner detector finds corners in the image using the description of the gradient distribution in a local neighborhood provided by the second moment matrix:

$M=\left[\begin{array}{cc}I_{x}^{2}(x, \sigma) & I_{x} I_{y}(x, \sigma) \\ I_{x} I_{y}(x, \sigma) & I_{y}^{2}(x, \sigma)\end{array}\right]$,

where $I(x, \sigma)$ is the derivative at position $x$ of the image smoothed with a Gaussian kernel of scale $\sigma$. From this matrix, the cornerness of a point can be computed using the following equation:

$R=\operatorname{Det}(M)-k \operatorname{Tr}(M)^{2}$, 


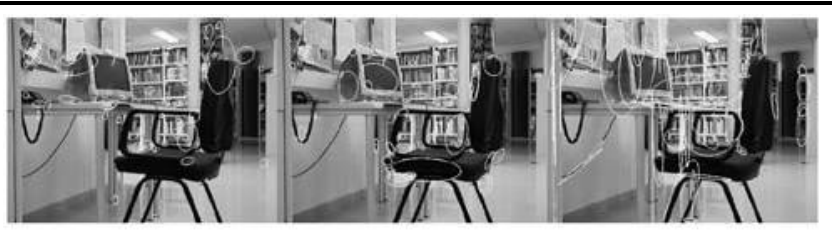

Fig. 1 Example of regions for the three affine covariant region detectors, from left to right: Harris-Affine, Hessian-Affine and MSER

where $k$ is a parameter usually set to 0.4 . Local maxima of this function are found across the scales, and the approach proposed by Lindeberg is used to select the characteristic scales.

Next, the parameters of an elliptical region are estimated minimizing the difference between the eigenvalues of the second order moment matrix of the selected region. This iterative procedure finds an isotropic region, which is covariant under affine transformations. The isotropy of the region is measured using the eigenvalue ratio of the second moment matrix:

$Q=\frac{\lambda_{\min }(\mu)}{\lambda_{\max }(\mu)}$,

where $Q$ varies from 1 for a perfect isotropic structure to 0 , and $\lambda_{\min }(\mu)$ and $\lambda_{\max }(\mu)$ are the two eigenvalues of the second moment matrix of the selected region at the appropriate scale. For a detailed description of this algorithm, the interested reader is referred to Mikolajczyk and Schmid (2004).

2. The Hessian-Affine detector is similar to the HarrisAffine, but the detected regions are blobs instead of corners. The base points are detected in scale-space as the local maxima of the determinant of the Hessian matrix:

$H=\left[\begin{array}{ll}I_{x x}(x, \sigma) & I_{x y}(x, \sigma) \\ I_{x y}(x, \sigma) & I_{y y}(x, \sigma)\end{array}\right]$

where $I_{x x}$ is the second derivative at position $x$ of the image smoothed with a Gaussian kernel of scale $\sigma$. The remainder of the procedure is the same as the HarrisAffine: base points are selected at their characteristic scales with the method proposed by Lindeberg and the affine shape of the region is found.

3. The Maximally Stable Extrema Regions (MSER) detector proposed by Matas et al. (2002) detects connected components where the intensity of the pixels is several levels higher or lower than the intensity of all the neighboring pixels of the region. Regions selected with this procedure may have an irregular shape, so the detected regions are approximated by an ellipse.

Because affine covariant regions must be compared, a common representation is necessary. Therefore all the regions detected with any method are normalized by mapping the detected elliptical area to a circle of a certain size. Once the affine covariant regions are detected and normalized, to reduce even more the effects caused by changes in the viewing conditions, these regions are characterized using a local descriptor. In our work, we have used Scale Invariant Feature Transform (SIFT) (Lowe 2004) and Gradient LocationOrientation Histogram (GLOH) (Mikolajczyk and Schmid 2005). These two descriptors were found to be the best in a comparison of various state of the art local descriptors (Mikolajczyk and Schmid 2005). The SIFT descriptor computes a 128 dimensional descriptor vector with the gradient orientations of a local region. In short, to construct the descriptor vector, the SIFT procedure divides the local region in 16 rectangular sub-regions and then, for every sub-region, it builds a histogram of 8 bins with the gradient orientations weighted with the gradient magnitude to suppress the flat areas with unstable orientations. The descriptor vector is obtained by concatenating the histograms for every sub-region.

The GLOH descriptor is similar to SIFT, with two main differences: the sub-regions are defined in a log-polar way, and the resulting descriptor vector has 272 dimensions but it is later reduced to 128 with a PCA.

These two descriptors are based on the same principle but with slightly different approaches. As they have no complementary properties, our objective in this comparison is to determine which one achieves the best performance. Therefore we have not combined them.

\section{Experimental design}

The objective of the present work is twofold: On the one hand, we want to validate the proposed method for indoor global localization and, on the other hand, we target to experimentally determine if using different region detectors simultaneously improves significantly the localization results. Although successive images acquired by the robot while moving in the room could be used to incrementally refine the localization, in our experiment, we wanted to evaluate if combining different region detectors improves the robustness to viewpoint change for the presented global localization method and therefore, we have only considered the worst case scenario, where only one image per room is available to localize the robot.

\subsection{Evaluation methodology}

In order to have a mapping technique that is scalable to large environments, it is important to have signatures as compact and resistant to viewpoint change as possible. However, there is no standard system to evaluate the robustness to viewpoint change of a topological location descriptor. Therefore, here we contribute a novel methodology to systematically evaluate the robustness to viewpoint change of 
omnidirectional location signatures as the ones proposed in this work.

We maintain that, although simple global localization experiments (i.e. determining the correct location of a new sensor reading in an already constructed map) are useful to assess the validity of a localization technique, it does not allow to compare different alternatives with the aim of finding those that favor smaller maps.

Our proposed approach to evaluating the robustness to viewpoint change of omnidirectional signatures consists in performing localization experiments in several sequences of panoramic images taken at fixed distance increments $(20 \mathrm{~cm}$ in this work) following a straight line predefined path.

Then, using the first panorama of every sequence as a node of the map, evaluate the remaining panoramas of each sequence to assess how distance affects the overall classification performance.

In order for the comparison to be significant, it is important that the dataset fulfills several conditions: In the first place, it must consist of a large number of different sequences in order to rule out the probability of randomly selecting the correct map node. In the dataset presented in this work we have used 17 map nodes, which stands for a $5.88 \%$ probability of randomly selecting the correct map node. Although this value is low, we believe it would be even better to reduce it to a value under $1 \%$ by adding more panorama sequences to the dataset. Next, it is important that the sequences have been acquired under a wide range of conditions in a variety of environments. The performance in large, well-textured rooms is typically better for appearance-based localization methods. Therefore, to enforce completeness of the comparison, the sequences dataset should contain various types of rooms.

Finally, the dataset used should be made publicly available in order to facilitate the validation of the results obtained and to allow comparison with other methods.

In this work we propose a dataset that accomplishes these three requirements. It consists of 17 sequences of panoramas from rooms in various buildings and has been made publicly available. ${ }^{1}$ In order to make the data set as general as possible, rooms with a wide range of characteristics have been selected (e.g., some sequences correspond to long and narrow corridors, while others have been taken in big hallways, large laboratories with repetitive patterns and others in smaller rooms such as individual offices). Panoramic images of the environment are shown Fig. 8 in the Appendix. A short description of each sequence is given below:

- iiia01 consists of 11 panoramas, and the sequence has been taken in a large robotics laboratory type of space.

\footnotetext{
${ }^{1}$ The data-set can be downloaded from http://www.iiia.csic.es/ aramisa.
}

- iiia02 and iiia03 are of 14 panoramas each, and have been taken at the conference room of the IIIA. In our experiments only the map node of iiia02 is used.

- iiia04 is 19 panoramas long, and has been acquired in a long and narrow corridor.

- iiia05 and iiia06 have 25 and 21 panoramas, respectively. They have been taken in the library of the IIIA, the first one is from the library entrance and librarian desk, while the second is from a narrow corridor with book shelves. Both share the first panorama of iiia05 as map node.

- iiia07 is 19 panoramas long. This represents another section of the robotics laboratory, and corresponds to a small cubicle.

- iiia08 is 10 panoramas long, and has been acquired in a small machinery room.

- iiia09 has 21 panoramas that have been taken at the back entrance hall. This sequence has been taken in a tilted floor, which is a challenge for the 4-point algorithm, because of the flat world assumption.

- iiia10 is 19 panoramas long and has been taken in the coffee room.

- iiia11 has 21 panoramas and has been acquired in the entrance hall of the IIIA.

- cvc01 is 21 panoramas long and corresponds to a long corridor of the CVC research center. As one of the corridor walls is made out of glass, the view field is wider than a normal corridor. However, direct sunlight affects the white balance of the image.

- cvc02 is 21 panoramas long, and has been acquired in a large office with many desks.

- cvc03 has 14 panoramas taken in a small office with just one working desk.

- cvc04 has 22 panoramas and has been taken in a wide corridor with posters.

- etse01 is the main hall of the engineering building and is 20 panoramas long.

- etse02 has 21 panoramas and has been taken in a very wide corridor of the engineering building.

\subsection{Panorama construction}

Instead of using an omnidirectional camera, the panoramas have been constructed by stitching together multiple views taken from a Sony DFW-VL500 camera mounted on a Directed Perception PTU-46-70 pan-tilt unit. The camera and pan-tilt unit can be seen in Fig. 2.

In order to build a panorama using a rotating camera, it had to be taken into consideration that the image sequence employed must have a fixed optical center. Translations of the optical center would introduce motion parallax, making the image sequence inconsistent. However, if the objects in the scene are sufficiently far from the camera, small translations can be tolerated. The steps to stitch all the images in a panorama are the following: 
1. The first step consists of projecting all the images of the sequence to a cylindrical surface. The points are mapped using the transformation from Cartesian to cylindrical coordinates:

$\theta=\tan ^{-1}\left(\frac{x}{f}\right), \quad v=\frac{y}{\sqrt{x^{2}+f^{2}}}$

where $x$ and $y$ are the position of the pixel, $f$ is the focal distance measured in pixels and $\theta$ and $v$ are respectively the angular position and the height of the point in the cylinder. The cylinder radius is the focal length of the camera used to acquire the images, as in this way the aspect ratio of the image is optimized (Shum and Szeliski 1997). Taking this into account, the size of the panoramas acquired by our system have a size of $5058 \times 500$ pixels.

2. Once all the images have been projected to cylindrical coordinates, the rotation between each pair of images must be estimated. In principle, only panning angles need to be recovered but, in practice, to correct vertical misalignment and camera twist, small vertical translations are allowed. Therefore, a displacement vector $\Delta t=\left(t_{x}, t_{y}\right)$ is estimated for every pair of input images. The implemented method to compute $\Delta t$ distinguishes between three situations:

i If sufficient feature points are found in the shared part of the images, $\Delta t$ is computed by means of matches between pairs of feature points. To find the translation with most support among matches, and to exclude false matches and outliers, RANSAC is used.

ii In those cases where there is not enough texture in the images to extract sufficient feature points, $\Delta t$ is computed looking for a peak in the normalized correlation between the edges detected by the Canny edge detector (Canny 1986) of the two images. This method has the advantage over other correlation-based approaches of being independent of the illumination conditions and the vignetting effect (intensity decreases towards the edge of the image). In addition, as all the image is used, even with small amounts of texture a reliable translation can be estimated. However, this technique is computationally more expensive than feature matching and is not invariant to rotations or other deformations in the image.

iii If no texture exists at all and the above procedure fails, the only remaining solution is to compute the expected translation if the angular displacement $\varphi$ (in radians) between the images is known: $t_{x}=f \varphi$ and $t_{y}=0$

3. Due to automatic camera gain, vignetting or radial distortion, an intensity jump may appear between two images as can be seen in Fig. 3. In this work the most straightforward solution is taken, that consists in blending lin-

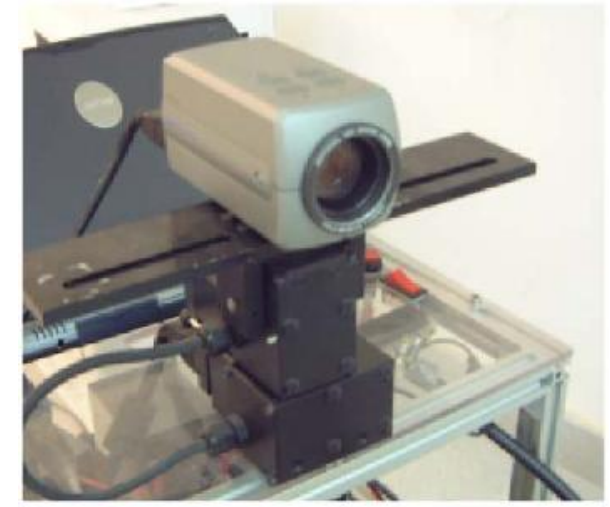

Fig. 2 The camera and pan-tilt unit used to take the images

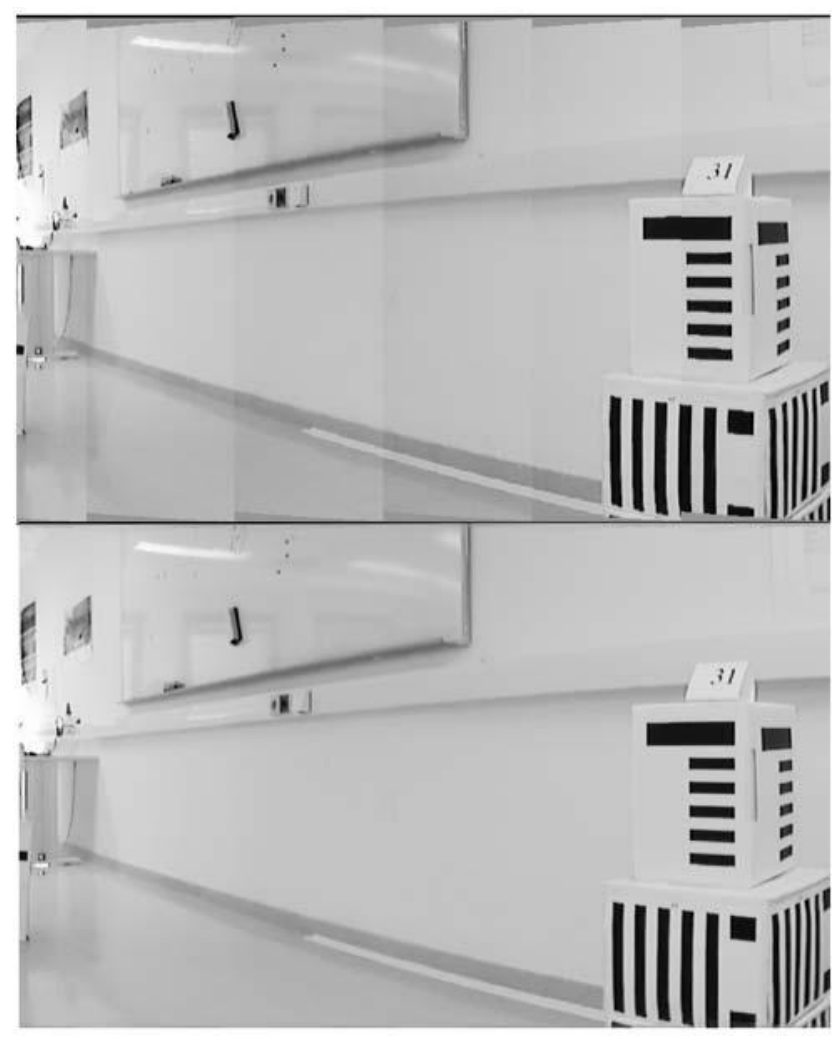

Fig. 3 Intensity jumps between successive images caused by automatic camera gain (top). Applying linear blending solves the problem (bottom)

early every two consecutive images. This method produces results good enough for visualization purposes and is suitable for static scenes. However techniques such as multi-band blending and deghosting as the ones proposed by Shum and Szeliski (1997), Brown and Lowe (2003), Szeliski and Shum (1997) or Uyttendaele et al. (2001) can be used to improve the result by eliminating stitching artifacts and dynamic objects that created ghosts in the panorama. 
Although the panoramic images were constructed for validation purposes, the constellations of feature region descriptors were not extracted from them. Instead, the features from the original images projected to cylindrical coordinates were used. The reason for this is to avoid false regions introduced by possible new artifacts created during the stitching process. The panoramas built with the stitching method were all correctly constructed, even in the case of changes in lightning, reflections, multiple instances of objects or lack of texture. The sequences have been acquired in uncontrolled environments.

\subsection{Panorama matching}

The region detectors and descriptors provided by Mikolajczyk et al. $(2005)^{2}$ were used to extract the affine-covariant regions from the images and compute the SIFT descriptor vectors.

The procedure to compare two panoramas is relatively straightforward. First, matches are established as nearest neighbors between the feature descriptors of both panoramas using the Euclidean distance as similarity measure. Potentially false matches are rejected comparing the distance of the first and the second nearest neighbor in the same way as proposed by Lowe (2004). Additionally, reciprocal matching is used to filter even more false matches: if feature $f_{a}$ from the first panorama matches feature $f_{b}$ of the second panorama, but feature $f_{b}$ does not match feature $f_{a}$, the match is discarded.

Next, the epipolar constraint between the panoramas is enforced by computing the essential matrix. The most straightforward way to automatically compute the essential matrix is using the normalized 8-point algorithm (Hartley and Zisserman 2004). However, assuming that the robot will only move through flat surfaces, it is possible to use a simplified version where only 4 correspondences are necessary.

$E=\left[\begin{array}{ccc}0 & e_{12} & 0 \\ e_{21} & 0 & e_{23} \\ 0 & e_{32} & 0\end{array}\right]$

Therefore, with a set of at least four correspondences of points of the form

$p=[x, y, z]=[\sin (2 \pi \tilde{x}), \tilde{y}, \cos (2 \pi \tilde{x})]$

where $\tilde{x}$ and $\tilde{y}$ are the normalized point coordinates in the planar panorama image, the following equations can be written:

$\left[\begin{array}{cccc}y_{1}^{\prime} x_{1} & x_{1}^{\prime} y_{1} & z_{1}^{\prime} y_{1} & y_{1}^{\prime} z_{1} \\ \vdots & \vdots & \vdots & \vdots \\ y_{n}^{\prime} x_{n} & x_{n}^{\prime} y_{n} & z_{n}^{\prime} y_{n} & y_{n}^{\prime} z_{n}\end{array}\right]\left[\begin{array}{c}e_{12} \\ e_{21} \\ e_{23} \\ e_{32}\end{array}\right]=0$

\footnotetext{
${ }^{2}$ http://www.robots.ox.ac.uk/ vgg/research/affine/.
}

where $\left(x_{i}, y_{i}, z_{i}\right)$ and $\left(x_{i}^{\prime}, y_{i}^{\prime}, z_{i}^{\prime}\right)$ is the $i^{\text {th }}$ pair of corresponding points. As outliers may still be present among the matches, RANSAC is used to automatically compute the essential matrix with most support. Finally, the set of inlier feature matches that agree with the epipolar constraint is used as the evidence of the relation between the two panoramas.

Given the high dimensionality of the feature descriptors, matching is expensive in terms of computational cost even for a small set of nodes. An alternative to exhaustive matching is to use a global similarity measure to re-rank the map nodes and estimate the essential matrix only for the $k$ top map nodes or, taking an any-time algorithm approach, until a node with a certain ratio of inliers is met. The global similarity measure should be fast to compute and exploit the differences between the map nodes to improve the reranking. We have applied the Vocabulary Tree proposed in Nister and Stewenius (2006) for object categorization to rerank the map nodes for a new query image as it fulfilled both requirements. In short, this method constructs a visual vocabulary tree of feature descriptors applying hierarchical $k$-means on a training dataset. Next, images are described as a normalized histogram of visual word counts. To give more emphasis to discriminative visual words, they are weighted using a Term Frequency-Inverse Document Frequency (TFIDF) approach. Finally, training set images can be re-ranked according to its Euclidean distance to the new image signature.

Although the presented method has a very good performance in our experiments, it is time-consuming to acquire a panorama rotating a pan-tilt unit every time a localization has to be performed. Instead, we evaluated the decrease in performance using uniquely a normal planar perspective image of $45^{\circ}$ field of view to localize the robot.

The simplest way to decide the corresponding node is by the maximum number of matches after computing the essential matrix (Valgren and Lilienthal 2008; Ramisa et al. 2008). An alternative we tried was to use the ratio between the number of matches and the lowest number of keypoints of the two images (Booij et al. 2007). Experimentally, we did not find much difference between both approaches in our dataset and therefore we have retained the first one.

\section{Experiments}

In order to achieve our two objectives, we tested all possible combinations of the three selected region detectors with two different descriptors. In addition to the listed region detectors we have evaluated the performance of the only scaleinvariant version of the Hessian Affine and Harris Affine detectors, but as the results obtained were significantly worse than the ones of the affine version are not displayed here. 
Table 1 Average percentage of correctly localized panoramas (acl) across all sequences and standard deviation (std). For convenience we have labeled M: MSER, HA: Harris-Affine, HE: Hessian-Affine, S: SIFT, G: GLOH

\begin{tabular}{|c|c|c|c|c|c|c|}
\hline \multirow[t]{2}{*}{ Combination } & \multicolumn{2}{|c|}{8 points algorithm } & \multicolumn{2}{|c|}{4 points algorithm } & \multicolumn{2}{|c|}{4 points and recipr. match } \\
\hline & acl & std & acl & std & acl & std \\
\hline $\mathrm{HA}+\mathrm{S}$ & $74 \%$ & $23 \%$ & $69 \%$ & $23 \%$ & $82 \%$ & $22 \%$ \\
\hline $\mathrm{HA}+\mathrm{G}$ & $70 \%$ & $21 \%$ & $73 \%$ & $24 \%$ & $81 \%$ & $21 \%$ \\
\hline $\mathrm{HE}+\mathrm{S}$ & $58 \%$ & $24 \%$ & $73 \%$ & $26 \%$ & $75 \%$ & $25 \%$ \\
\hline $\mathrm{HE}+\mathrm{G}$ & $63 \%$ & $26 \%$ & $65 \%$ & $27 \%$ & $74 \%$ & $26 \%$ \\
\hline $\mathrm{M}+\mathrm{S}$ & $62 \%$ & $28 \%$ & $78 \%$ & $18 \%$ & $76 \%$ & $23 \%$ \\
\hline $\mathrm{M}+\mathrm{G}$ & $61 \%$ & $29 \%$ & $69 \%$ & $23 \%$ & $74 \%$ & $26 \%$ \\
\hline $\mathrm{HA}+\mathrm{HE}+\mathrm{S}$ & $64 \%$ & $15 \%$ & $78 \%$ & $19 \%$ & $86 \%$ & $14 \%$ \\
\hline $\mathrm{HA}+\mathrm{HE}+\mathrm{G}$ & $67 \%$ & $14 \%$ & $79 \%$ & $21 \%$ & $87 \%$ & $16 \%$ \\
\hline $\mathrm{M}+\mathrm{HE}+\mathrm{S}$ & $56 \%$ & $23 \%$ & $75 \%$ & $23 \%$ & $87 \%$ & $15 \%$ \\
\hline $\mathrm{M}+\mathrm{HE}+\mathrm{G}$ & $60 \%$ & $23 \%$ & $78 \%$ & $18 \%$ & $88 \%$ & $14 \%$ \\
\hline $\mathrm{M}+\mathrm{HA}+\mathrm{S}$ & $65 \%$ & $21 \%$ & $79 \%$ & $19 \%$ & $86 \%$ & $14 \%$ \\
\hline $\mathrm{M}+\mathrm{HA}+\mathrm{G}$ & $70 \%$ & $25 \%$ & $79 \%$ & $19 \%$ & $88 \%$ & $11 \%$ \\
\hline $\mathrm{M}+\mathrm{HA}+\mathrm{HE}+\mathrm{S}$ & $62 \%$ & $16 \%$ & $82 \%$ & $19 \%$ & $89 \%$ & $11 \%$ \\
\hline $\mathrm{M}+\mathrm{HA}+\mathrm{HE}+\mathrm{G}$ & $64 \%$ & $20 \%$ & $82 \%$ & $19 \%$ & $90 \%$ & $11 \%$ \\
\hline
\end{tabular}

Table 1, shows the average percentage of correctly classified test panoramas for each combination. Results are provided using the 8-point algorithm, the 4-point algorithm and also the later with reciprocal matches. From the results illustrated in the table for the 4 point algorithm with reciprocal matches, it can be seen that by reducing the number of false matches with the reciprocal matches technique, improves substantially the performance. Therefore from now on, we only show the results obtained with this technique. The average percentage of correct localizations has been computed by first computing the percentage of correctly classified panoramas for each sequence individually and then computing the mean across all the sequences.

Standard deviation is also provided in order to assess the stability of combinations along the different sequences. The high standard deviation is mainly due to bad results of the combinations in particular sequences. Not much difference is observed among the descriptors GLOH and SIFT, which performed similarly in all cases. Looking at the feature detectors individually, the best results have been obtained by Harris Affine, while Hessian Affine and MSER had a similar performance.

Overall, the combinations of detectors outperformed the individual detectors. The best performance in the localization test has been achieved by the combination of the three detectors, which classified correctly $90 \%$ of the panoramas. This performance is mainly due to their good complementarity. In Fig. 4 the average performance of two selected combinations is compared to the standalone detectors with the proposed evaluation methodology to evaluate the robustness of the methods to viewpoint change.

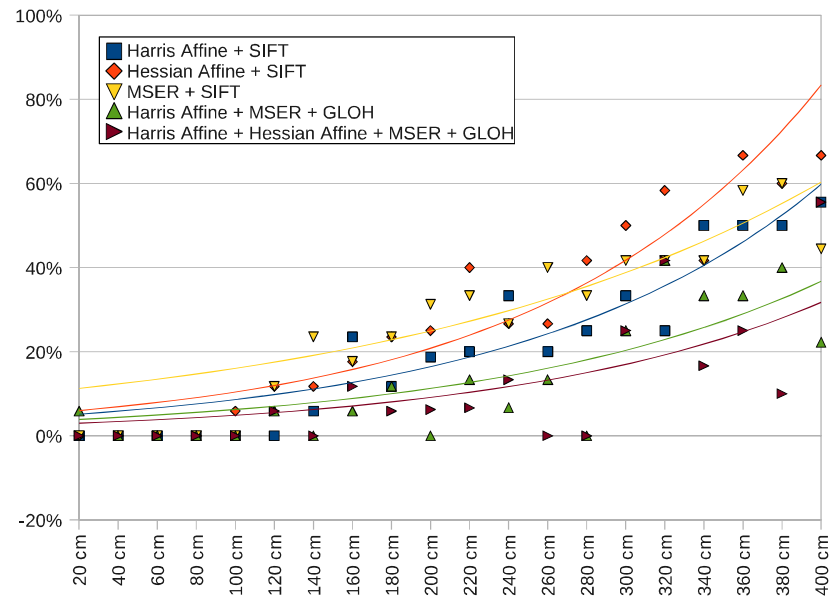

Fig. 4 Percentage of incorrectly classified test panoramas as a function of the distance in centimeters to the map node (i.e. first panorama of the sequence). The exponential regression of the data points is also provided for clarity. Best viewed in color

As can be seen, the combinations obtained in the order of $20 \%$ more correct localization at four meters according to the estimated exponential regression. Sequences acquired in large rooms typically achieved a good performance no matter the combination used. However, small rooms and specially long and narrow corridors seem to be more difficult environments, even if they are well textured. This can be explained because the distance between the robot and the perceived objects is short and, therefore, the objects' appearance changes rapidly resulting in an unreliable matching in the lateral regions of the panorama. 
Table 2 Average percentage of correctly localized panoramas for some interesting sequences. The naming convention is the same as in Table 1

\begin{tabular}{lcccc}
\hline Combination & cvc01 & iiia04 & iiia06 & iiia09 \\
\hline HA+S & $35 \%$ & $42 \%$ & $75 \%$ & $75 \%$ \\
HA+G & $35 \%$ & $47 \%$ & $70 \%$ & $70 \%$ \\
HE+S & $20 \%$ & $47 \%$ & $75 \%$ & $45 \%$ \\
HE+G & $20 \%$ & $53 \%$ & $80 \%$ & $30 \%$ \\
M+S & $85 \%$ & $42 \%$ & $30 \%$ & $65 \%$ \\
M+G & $95 \%$ & $53 \%$ & $35 \%$ & $35 \%$ \\
HA+HE+S & $80 \%$ & $84 \%$ & $70 \%$ & $95 \%$ \\
HA+HE+G & $45 \%$ & $89 \%$ & $75 \%$ & $85 \%$ \\
M+HE+S & $90 \%$ & $84 \%$ & $80 \%$ & $65 \%$ \\
M+HE+G & $90 \%$ & $84 \%$ & $90 \%$ & $60 \%$ \\
M+HA+S & $90 \%$ & $79 \%$ & $70 \%$ & $85 \%$ \\
M+HA+G & $90 \%$ & $89 \%$ & $65 \%$ & $80 \%$ \\
M+HA+HE+S & $80 \%$ & $95 \%$ & $75 \%$ & $80 \%$ \\
M+HA+HE+G & $75 \%$ & $100 \%$ & $90 \%$ & $75 \%$ \\
\hline
\end{tabular}

Some particularly difficult sequences have been $\mathrm{cvc} 01$, iiia04, iiia06 and iiia09. Table 2 shows the results with these sequences. As we can see, the performance is notably increased by combining the strengths and weaknesses of the different detectors. On average, standalone detectors achieved around 55\%, while combinations increased to around $81 \%$ in these environments. Sequence iiia04 is long and narrow corridor. In this environment only features found in the antipodal points of the panorama that correspond to the movement direction are reliable, since the other are affected by an extreme appearance variance due to the large point of view change (i.e. the closer the object, the larger the point of view change for the same traveled distance). Therefore, combining various detectors increases the number of matches and the robustness of the computed essential matrix. It is interesting to notice that in this case the combination of all feature types achieved $100 \%$ correct classification.

The iiia06 is also a long and narrow corridor, but in this case much more texture is present. In consequence, the Harris Affine and the Hessian Affine detectors alone find enough reliable features to correctly estimate the essential matrix.

Another notable finding is the extremely good performance of MSER on cvc01 when compared to the other detectors. The explanation for the good performance of the MSER in this sequence is due to the robustness of this detector to extreme intensity variations: The MSER detector reacts to high contrast regions, that are preserved even under sunlight overexposure.

Most of the similar approaches to global localization (e.g. Booij et al. 2007) use feature detectors only invariant to scale but not affine covariant, mainly because of its more
Table 3 Average feature matching and RANSAC time per map node. It is important to notice the difference in time scale

\begin{tabular}{lll}
\hline Combination & $\begin{array}{l}\text { Matching } \\
\text { (seconds) }\end{array}$ & $\begin{array}{l}\text { RANSAC } \\
\text { (milliseconds) }\end{array}$ \\
\hline $\mathrm{HA}+\mathrm{S}$ & 4.31 & 3.046 \\
$\mathrm{HA}+\mathrm{G}$ & 4.29 & 2.597 \\
$\mathrm{HE}+\mathrm{S}$ & 2.87 & 3.016 \\
$\mathrm{HE}+\mathrm{G}$ & 2.88 & 2.631 \\
$\mathrm{M}+\mathrm{S}$ & 1.24 & 2.920 \\
$\mathrm{M}+\mathrm{G}$ & 1.24 & 2.310 \\
$\mathrm{HA}+\mathrm{HE}+\mathrm{S}$ & 7.16 & 6.625 \\
$\mathrm{HA}+\mathrm{HE}+\mathrm{G}$ & 7.16 & 5.401 \\
$\mathrm{M}+\mathrm{HE}+\mathrm{S}$ & 4.11 & 5.827 \\
$\mathrm{M}+\mathrm{HE}+\mathrm{G}$ & 4.11 & 5.361 \\
$\mathrm{M}+\mathrm{HA}+\mathrm{S}$ & 5.51 & 6.682 \\
$\mathrm{M}+\mathrm{HA}+\mathrm{G}$ & 5.51 & 5.382 \\
$\mathrm{M}+\mathrm{HA}+\mathrm{HE}+\mathrm{S}$ & 8.44 & 1.3941 \\
$\mathrm{M}+\mathrm{HA}+\mathrm{HE}+\mathrm{G}$ & 8.47 & 1.0815 \\
\hline
\end{tabular}

expensive computational cost. For comparability, we have evaluated the performance of the Difference of Gaussians detector (Lowe 2004). This method uses as initial points the local maxima of the Differences of Gaussians (DoG), defines a circular region around these initial points and finally SIFT is used to describe the selected regions. For our tests we used the implementation provided by Lowe. ${ }^{3}$ Using points detected with the DoG and SIFT, the average correct location was $72 \%$. However, it had an irregular performance depending on the environment type (27\% standard deviation), with perfect results in large rooms, but very poor results in narrow corridors and small rooms. This was an expected outcome as this detector is less resistant to viewpoint changes.

In terms of computational complexity, the most expensive step of the approach is clearly the bidirectional descriptor matching as can be seen in Table 3. These computational times have been obtained with a $\mathrm{C}++$ implementation of the method running in a Linux Pentium 4 at $3.0 \mathrm{GHz}$ computer with $2 \mathrm{~Gb}$ of RAM.

\subsection{Re-ranking of map nodes}

As explained in Sect. 4.3, the global appearance based image similarity measure from Nister and Stewenius has been used to re-rank the map nodes and prioritize those that appear more similar. We have build the vocabulary tree with Harris Affine features. When used for object classification, this type of approach requires at least tens of training images in order to correctly determine the class of a novel ob-

\footnotetext{
${ }^{3}$ http://www.cs.ubc.ca/ lowe/keypoints/.
} 


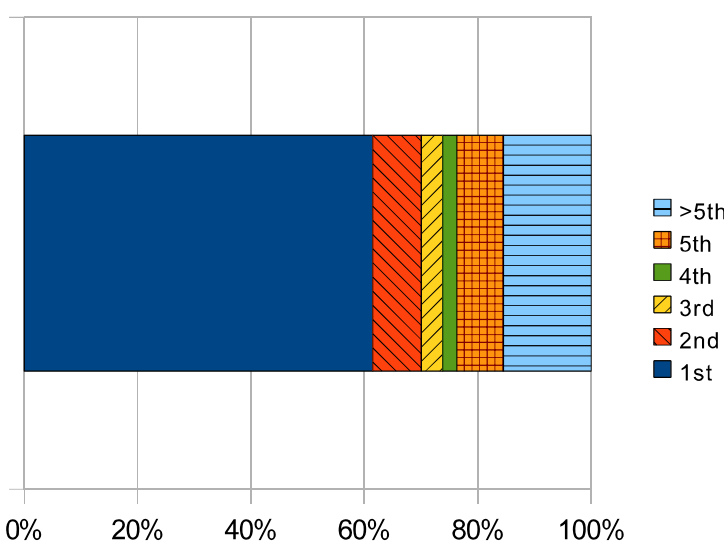

Fig. 5 Position of the correct map node after re-ranking using the vocabulary tree

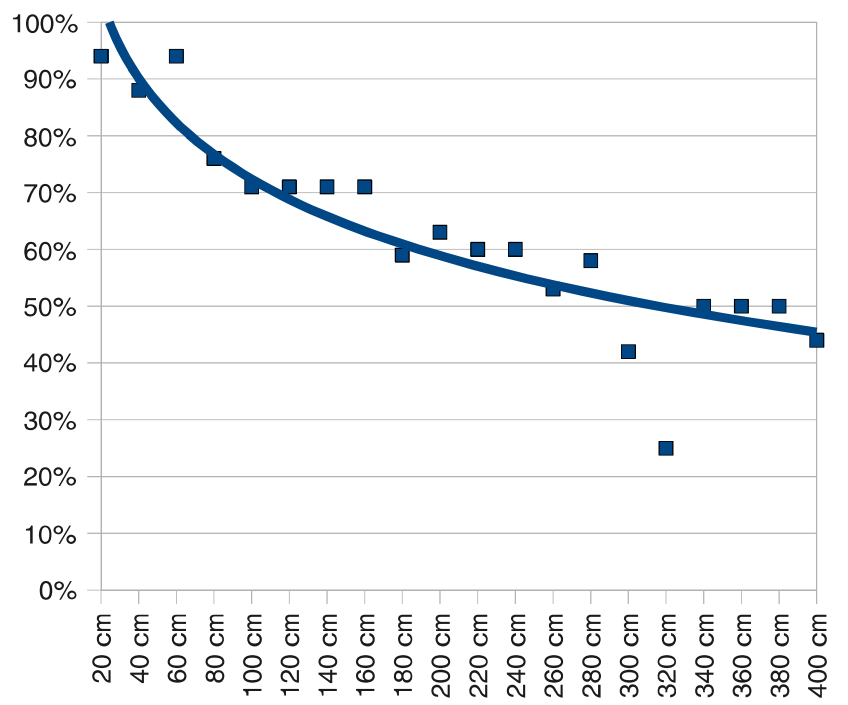

Fig. 6 Ratio of query images with the correct node re-ranked at the top position against distance to the map node (i.e. first panorama of the sequence). The logarithmic regression curve is also shown

ject instance. However, we only used the map nodes to train both the vocabulary tree and the classifier. This gives only one training instance for each class. Despite so limited training data, the approach achieved the notable overall result of re-ranking the correct node in the first position for $62 \%$ of the query panoramas, and among the top five nodes $85 \%$ of times as can be seen in Fig. 5. More detailed results of this re-ranking experiment are in Fig. 7, where the performance is shown for each individual sequence.

As expected, the percentage of times the correct map node is re-ranked at the top position decreases as the physical distance to the query panorama increases (see Fig. 6).

\subsection{Localization with $45^{\circ} \mathrm{FOV}$ images}

Constructing a panoramic image with a rotating camera on a pan-tilt unit is a time-consuming step that requires the ro- 1st $\triangle$ 2nd $\square$ 3rd $\square$ 4th $\otimes$ 5th $\boxminus>5$ th

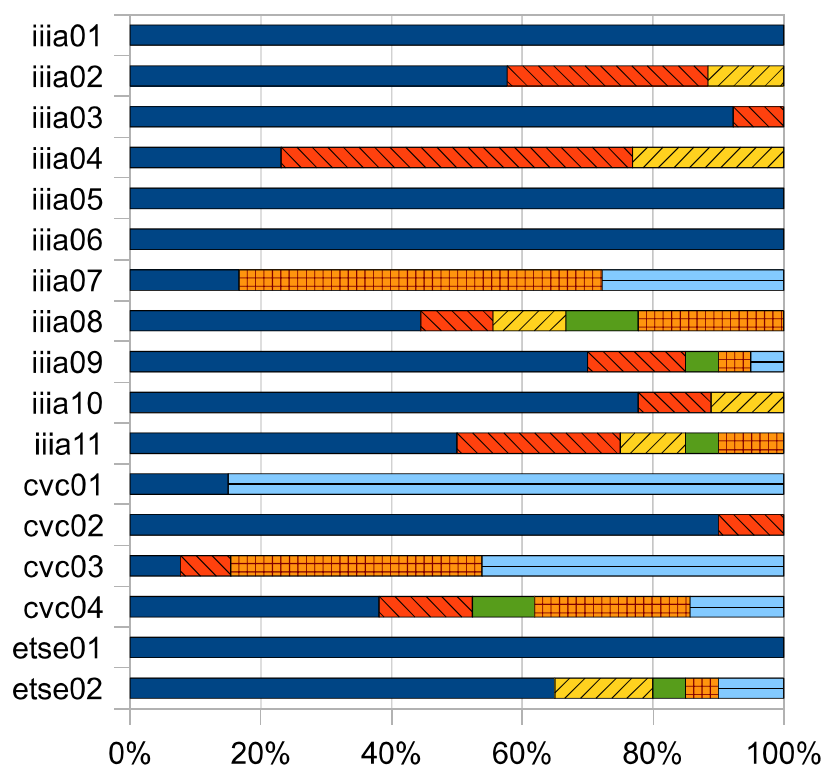

Fig. 7 Position of the correct map node after re-ranking using the vocabulary tree per sequence

bot to stay in a fixed position during the acquisition. In order to assess the decrease in performance that would cause using just a single conventional image to localize the robot we have done the following experiment: For every test panorama, a random area that spans $45^{\circ}$ and has at least 100 features is extracted and matched to the map nodes. This procedure is repeated for every test panorama. After a 10 repetitions experiment with all test panoramas, the average number of correct localizations was $73 \%$ using Harris Affine combined with MSER and the GLOH descriptor. This result is good considering how limited the field of view is. In addition to the time saved in image acquisition, the matching time is reduced almost one order of magnitude on average.

\section{Conclusions and discussion}

In this work we have proposed and evaluated a signature to characterize places that can be used for global localization. This signature consists of a constellation of feature descriptors, computed from affine-covariant regions, extracted from a panoramic image, that has been acquired in the place we want to add to the map. Later, these signatures are compared to the constellation extracted from a new panoramic image using geometric constraints, and the most similar signature is selected as the current location. To compare the different signatures, the 4-point algorithm with bidirectional matching and RANSAC to reject false matches are used. Results obtained with the current approach clearly surpass the ones obtained in earlier work using the 8-point algorithm (Ramisa et al. 2008) and the 4-point algorithm without 
bidirectional matching. Combinations of feature detectors have been shown to perform best if combined with adequate mechanisms, such as the aforementioned reciprocal matching or distance to the second nearest neighbor, to reject incorrect pairings of features before computing the essential matrix.

Furthermore, we have proposed an evaluation strategy to assess the robustness to change in point of view for appearance-based signatures used for global localization in a topological map. This aims to help finding more robust signatures that will yield more compact and scalable maps.

When applying proposed evaluation method to our global localization schema, the results obtained show that by using the combination of different feature detectors, a room can be reliably recognized in indoor environments from a distance of up to 4 meters from the point where the reference panorama was obtained. The best results $(90 \%$ correct localizations) were achieved by combining all three detectors.

Moreover, we have also compared the results of our proposed affine-covariant region detectors approach with the scale-invariant region detectors methodology proposed in Lowe (2004), widely used in robot navigation, and showed that the affine-covariant regions outperformed Lowe's scaleinvariant method.

In order to speed-up the otherwise very expensive descriptor matching phase, a global similarity technique usually employed for object recognition, the vocabulary tree of Nister and Stewenius (2006), has been effectively applied to re-rank the map nodes for a given query panorama and save most of the computation time.

Furthermore, we tested how the performance degrades if only a conventional perspective image is used instead of an omnidirectional image. Results of a 10 repetitions experiment with random $45^{\circ}$ sections (with a minimum amount of texture) from all the test panoramas showed a surprisingly good performance.

\section{Appendix}

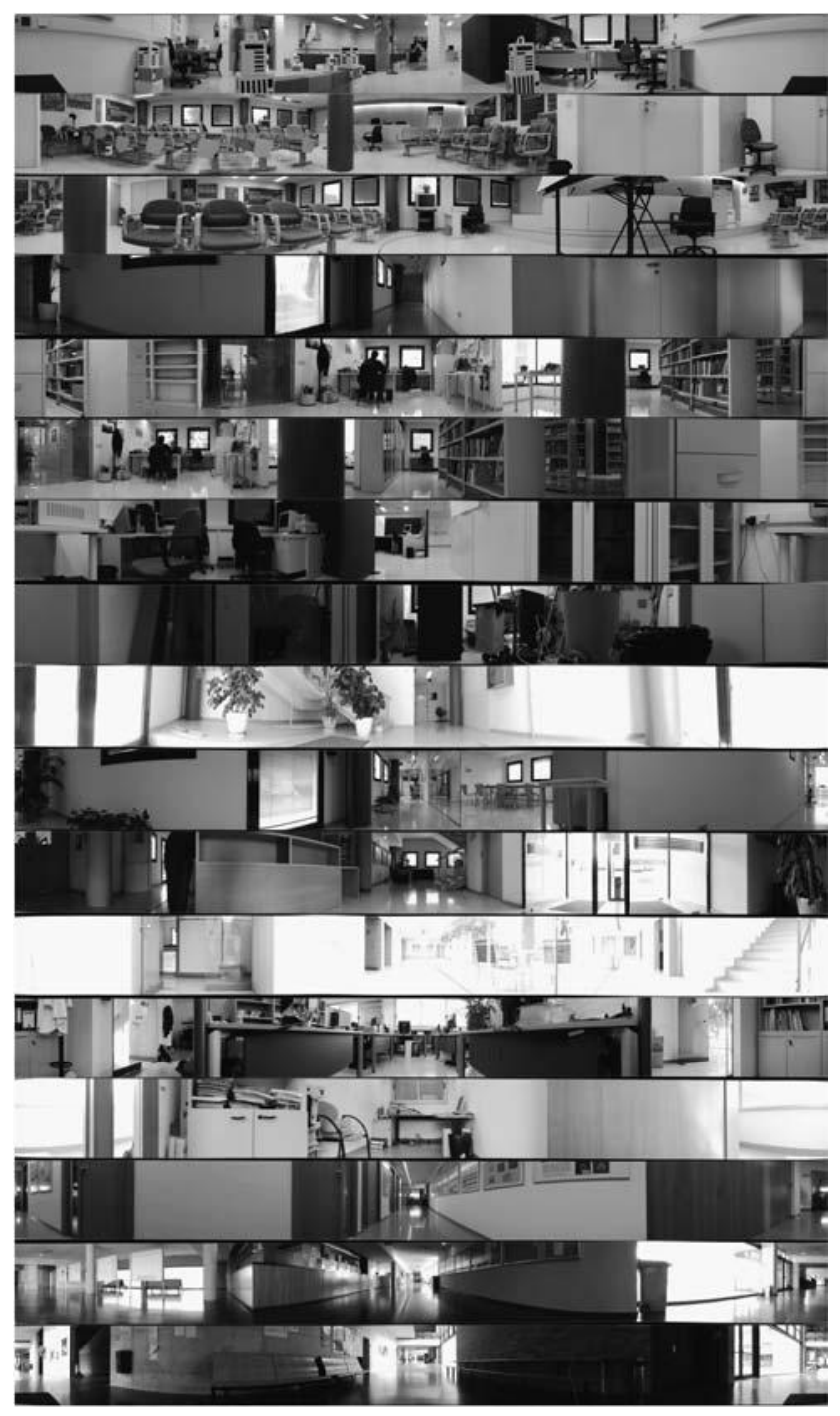

Fig. 8 Panorama nodes in the same order as described in the text

\section{References}

Andreasson, H., Treptow, A., \& Duckett, T. (2005). Localization for mobile robots using panoramic vision, local features and particle filters. In: Proceedings of the IEEE international conference on robotics and automation (ICRA'05), Barcelona, Spain.

Angeli, A., Filliat, D., Doncieux, S., \& Meyer, A. J. (2008). A fast and incremental method for loop-closure detection using bags of visual words. IEEE Transactions on Robotics, Special Issue on Visual SLAM.

Bay, H., Ess, A., Tuytelaars, T., \& Gool, L. V. (2008). Surf: Speeded up robust features. Computer Vision and Image Understanding, $110(3), 346-359$. 
Beeson, P., Jong, K. N., \& Kuipers, B. (2005). Towards autonomous topological place detection using the extended Voronoi graph. In: IEEE international conference on robotics and automaton (ICRA), Barcelona, Spain, pp. 4373-4379.

Booij, O., Terwijn, B., Zivkovic, Z., \& Krose, B. (2007). Navigation using an appearance based topological map. In: IEEE international conference on robotics and automation (ICRA), pp. 3927-3932.

Brown, M., \& Lowe, D. (2003). Recognising panoramas. In ICCV'03: Proceedings of the ninth IEEE international conference on computer vision (p. 1218). Washington: IEEE Computer Society.

Canny, J. (1986). A computational approach to edge detection. IEEE Transactions on Pattern Analysis and Machine Intelligence, 8(6), 679-698.

Castellanos, A. J., \& Tardos, D. J. (1999). Mobile robot localization and map building: Multisensor fusion approach. Dordrecht: Kluwer.

Choset, H., \& Nagatani, K. (2001). Topological simultaneous localization and mapping (SLAM): Toward exact localization without explicit localization. IEEE Transactions on Robotics and Automation, 17(2), 125-137.

Cummins, M., \& Newman, P. (2008). Accelerated appearance-only SLAM. In: Robotics and automation, 2008. ICRA 2008. IEEE international conference on, pp. 1828-1833.

Dissanayake, M., Newman, P., Clark, S. M., Durrant-Whyte, H., \& Csorba, M. (2001). A solution to the simultaneous localization and map building (slam) problem. IEEE Transactions on Robotics and Automation, 17(3), 229-241.

Franz, M., Schlkopf, O., Mallot, B., \& Blthoff, A. H. (1998). Learning view graphs for robot navigation. Autonomous Robots, 5, 111125.

Hartley, R., \& Zisserman, A. (2004). Multiple view geometry in computer vision, 2nd edn. Cambridge: Cambridge University Press, ISBN: 0521540518.

Lindeberg, T. (1998). Feature detection with automatic scale selection. International Journal of Computer Vision, 30(2), 79-116.

Lowe, D. (2004). Distinctive image features from scale-invariant keypoints. International Journal of Computer Vision, 60(2), 91-110.

Matas, J., Chum, O., Urban, M., \& Pajdla, T. (2002). Robust wide baseline stereo from maximally stable extremal regions. In: Proceedings of the British machine vision conference (BMVC'02), Cardiff, UK

Mikolajczyk, K., \& Schmid, C. (2004). Scale \& affine invariant interest point detectors. International Journal of Computer Vision, 60(1), 63-86.

Mikolajczyk, K., \& Schmid, C. (2005). A performance evaluation of local descriptors. IEEE Transactions on Pattern Analysis \& Machine Intelligence, 27(10), 1615-1630

Mikolajczyk, K., Tuytelaars, T., Schmid, C., Zisserman, A., Matas, J., Schaffalitzky, F., Kadir, T., \& van Gool, L. (2005). A comparison of affine region detectors. International Journal of Computer Vision, 65(2), 43-72.

Nister, D., \& Stewenius, H. (2006). Scalable recognition with a vocabulary tree. Conf. Computer Vision and Pattern Recognition, 2 , 2161-2168.

Owen, C., \& Nehmzow, U. (1998). Landmark-based navigation for a mobile robot. In From animals to animals: Fifth international conference on simulation of adaptive behavior (SAB) (pp. 240245). Cambridge: MIT

Ramisa, A., Tapus, A., Lopez de Mantaras, R., \& Toledo, R. (2008). Mobile robot localization using panoramic vision and combinations of feature region detectors. In: Robotics and automation, 2008. ICRA 2008. IEEE international conference on, pp. 538543.

Se, S., Lowe, D., \& Little, J. (2002). Mobile robot localization and mapping with uncertainty using scale-invariant visual landmarks. International Journal of Robotics Research, 21(8), 735-758.
Shum, H., \& Szeliski, R. (1997). Panoramic image mosaics. Tech. Rep. MSR-TR-97-23, Microsoft Research.

Silpa-Anan, C., \& Hartley, R. A. (2004). Localization using an imagemap. In: Proceedings of the 2004 Australasian conference on robotics and automation, Canberra, Australia.

Szeliski, R., \& Shum, H. Y. (1997). Creating full view panoramic image mosaics and environment maps. In SIGGRAPH '97: Proceedings of the 24th annual conference on computer graphics and interactive techniques, vol. 31 (pp. 251-258). New York: ACM/Addison-Wesley.

Tapus, A., \& Siegwart, R. (2006). A cognitive modeling of space using fingerprints of places for mobile robot navigation. In: Proceedings IEEE international conference on robotics and automation (ICRA), Orlando, USA, pp. 1188-1193.

Thrun, S. (1998). Learning metric-topological maps for indoor mobile robot navigation. Artificial Intelligence, 99(1), 21-71.

Thrun, S. (2000). Probabilistic algorithms in robotics. Artificial Intelligence Magazine, 21, 93-109.

Uyttendaele, M., Eden, A., \& Szeliski, R. (2001). Eliminating ghosting and exposure artifacts in image mosaics. In: IEEE computer society conference on computer vision and pattern recognition. IEEE Computer Society, vol. 2.

Valgren, C., \& Lilienthal, A. (2008). Incremental spectral clustering and seasons: Appearance-based localization in outdoor environments. In: Robotics and automation, 2008. ICRA 2008. IEEE international conference on, pp. 1856-1861.

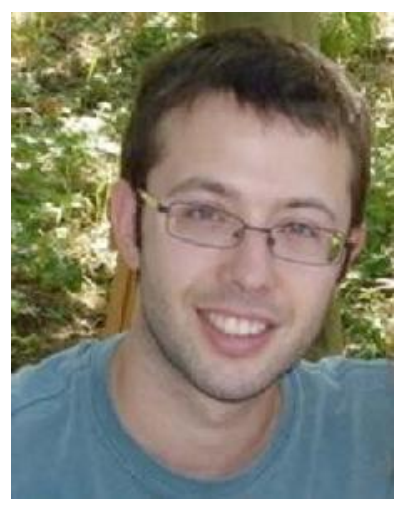

Arnau Ramisa is a Ph.D. student in Computer Vision and Artificial Intelligence at the Artificial Intelligence Research Institute (IIIA, CSIC). He obtained his M.Sc. in Computer Science from the $\mathrm{Au}$ tonomous University of Barcelona, Spain, in 2006 and his degree of Engineer in Computer Science from the Autonomous University of Barcelona in 2004. His research interests are mainly focused in computer vision, vision-based robot localization, real-time object recognition, human-machine interaction and machine learning.

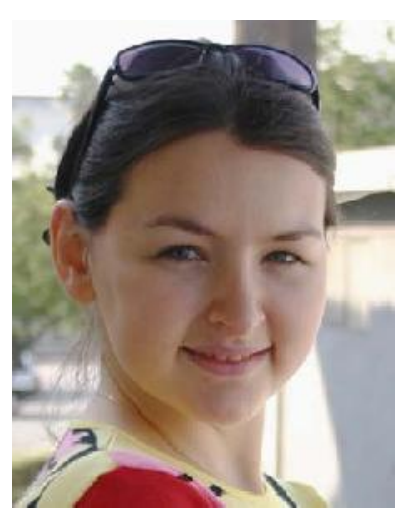

Adriana Tapus is currently an Assistant Professor at ENSTAParisTech (Paris, France), in the Cognitive Robotics Lab, Computer Science Department. She conducted postdoctoral research from 2005 to 2009 in the Interaction Lab, at the University of Southern California, USA. She received her Ph.D. in Computer Science from Swiss Federal Institute of Technology, Lausanne (EPFL) in 2005, her M.Sc. in Computer Science from University Joseph Fourier, Grenoble, France in 2002 and her degree of Engineer in Computer Science and Engineering from Politehnica University of Bucharest, Romania in 2001. Her Ph.D. dissertation pertained to finding a natural solution to do humanlike navigation using fingerprints of places. Her current research interests include socially assistive robotics, human-robot interaction, humanoid robotics, machine learning, and computer vision. At http://www.ensta.fr/ tapus research details can be found. 


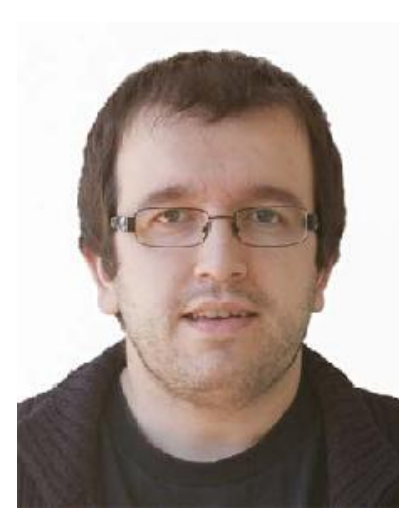

David Aldavert is a $\mathrm{Ph} . \mathrm{D}$. student in Computer Vision and Robotics at the Computer Vision Center of the Autonomous University of Barcelona. He obtained his M.Sc. in Computer Science from the Autonomous University of Barcelona, Spain, in 2006 and his degree of Engineer in Computer Science from the same university in 2004. His research interests are mainly focused in computer vision, visual SLAM, loop closure and sensor fusion.

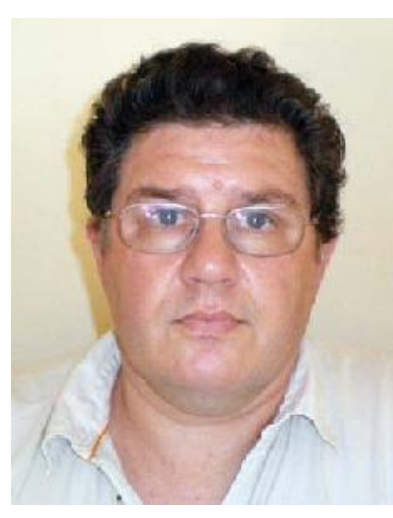

Ricardo Toledo received the degree in Electronic Engineering from the Universidad Nacional de Rosario (Argentina) in 1986, the M.Sc. degree in image processing and artificial intelligence from the Universitat Autònoma de Barcelona (UAB) in 1992 and the Ph.D. in 2001. Since 1989 he has been giving lectures at the Computer Science Dpt. of the UAB and participating in $\mathrm{R}+\mathrm{D}$ projects. Currently he is a full time associated professor. In 1996 he participated in the foundation of the Computer Vision Center (CVC) at the UAB, presently being the responsible of the research group on autonomous robots. Ricardo has participated in national and international
$\mathrm{R}+\mathrm{D}$ projects being the leader of some of them, and is coauthor of more than 30 papers, all these in the field of computer vision, robotics and medical imaging.

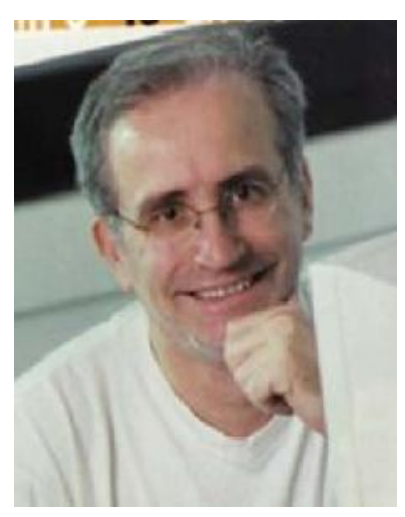

Ramon Lopez de Mantaras Research Professor and Director of the Artificial Intelligence Research Institute of the Spanish National Research Council. Master of Sciences in Computer Science from the University of California Berkeley, $\mathrm{Ph} . \mathrm{D}$. in Physics from the University of Toulouse, and Ph.D. in Computer Science from the Technical University of Barcelona. A pioneer of Artificial Intelligence in Spain, with contributions, since 1976, in Unsupervised Learning, Pattern Classification, Approximate Reasoning, Expert Systems, Inductive Learning, Case-Based Reasoning, Autonomous Robots, AI \& Music, and Bayesian Learning. Author of over 220 papers. Invited plenary speaker in numerous international conferences. Former Editor-in-Chief of Artificial Intelligence Communications, editorial board member of several international journals including the Autonomous Robots Journal and AI Magazine. Associate Editor of the Artificial Intelligence Journal. Program committee Chairman of UAI-94 and ECML'00. Conference Chairman of ECAI-06, ECML07 and IJCAI-07. ECCAI Fellow and recipient of numerous awards including four best paper awards at international conferences, the Digital European AI research award, and the "City of Barcelona" Research Prize. President of the Board of Trustees of IJCAI. For additional information please visit the web site: http://www.iiia.csic.es/ mantaras. 